\title{
Reflexiones sobre la escuela rural. Un modelo educativo de éxito
}

Reflections on the rural school. A successful educational model

Joan Tahull Fort

e-mail: joantfort@geosoc.udl.cat

Universitat de Lleida. España

Iolanda Montero Plaza

e-mail:ymontero@xtec.cat

Departement d'Ensenyament de la Generalitat de Catalunya. España

\section{Resumen}

En el presente artículo se describe y analiza la escuela rural actual en Cataluña. Se presentan datos comparativos de los resultados académicos de las pruebas de competencias básicas de los alumnos de $6^{\circ}$ de Primaria de las escuelas rurales y del global de la Comunidad Autónoma, de tres cursos consecutivos. Se describen algunas de sus potencialidades y fortalezas. Para la realización de la investigación se han utilizado diferentes datos oficiales de la Generalitat de Cataluña y también se han entrevistado a maestros/as, padres/madres y exalumnos/as todos relacionados con la escuela rural. Tradicionalmente la escuela rural en España, también en Cataluña, era maltratada, descuidada y muchas veces abandonada. Esta enseñanza era una copia imperfecta y deformada de la urbana. En la actualidad la escuela rural ha mejorado significativamente los resultados académicos y en valoración y respeto por el trabajo realizado. La organización está consolidada, tiene éxito y no está cuestionada. Quizás la escuela rural deba guiar y orientar la escuela urbana hacia una educación más dinámica, flexible y personal.

Palabras clave: pueblo; ruralidad; educación; prestigio; alumno.

\begin{abstract}
This article describes and analyzes the current rural school in Catalonia. We present comparative data of the academic results of the tests of basic competences of the students of Primary of 6 of the rural schools and of the global of the Autonomous Community. It describes some of its potentialities and strengths. In order to carry out the research, different official data of the Generalitat of Catalonia have been used, and in-depth interviews have also been conducted with teachers, parents and alumni all related to the rural school. Traditionally the rural school in Spain, also in Catalonia, was mistreated, neglected and often abandoned. This teaching was an imperfect and deformed copy of the urban one. At present the rural school has improved significantly in the academic results and in appreciation and respect for the work done. The organization is consolidated, successful and unquestioned. Perhaps the rural school should guide and orient the urban school towards a more dynamic, flexible and personal education.
\end{abstract}

Keywords: village; rurality; education; prestige; student.

Cómo referenciar este artículo / How to reference this article:

Tahull, J., \& Montero, I. (2018). Reflexiones sobre la escuela rural. Un modelo educativo de éxito.

Tendencias Pedagógicas, 32, 161-176. doi: 10.15366/ tp2018.32.012 


\section{Introducción}

La escuela rural en España, también en Cataluña, ha sido tradicionalmente maltratada, descuidada y demasiadas veces abandonada. El sistema educativo español desde el siglo XIX ha sido centralizado y uniformizado. Estos principios han marcado históricamente la escuela rural, sin tener en cuenta sus particularidades sociales y culturales muy diferentes de los centros ubicados en entornos urbanos. La ley Moyano de 1857 estableció la obligatoriedad de tener escuela en las poblaciones de más de 500 habitantes sin dotarlas de los recursos adecuados: muchas eran incompletas, los locales eran deficientes, condiciones de trabajo inadecuadas y formación escasa del maestroํ. En 1900 se constituyó el Ministerio de Instrucción Pública para organizar y gestionar la educación española, tuvo en general un impacto positivo en la escuela, aunque su incidencia en la escuela rural fue escasa y siguió estancada y abandonada (Soler, 2008). El maestro rural era un docente incompleto en una escuela defectuosa, muy lejos en calidad pedagógica de los centros educativos urbanos. Estos enseñantes tenían una formación inicial deficiente o nula, un sueldo mínimo, una consideración social baja, unas expectativas alejadas del entorno rural y muchos vivían aislados en pueblos desconocidos para ellos. Los resultados educativos eran pobres, no se ofrecía una enseñanza idónea. Durante mucho tiempo estas características impregnaban las escuelas rurales españolas, también catalanas. Estos centros eran predominantemente unitarios, tenía un número reducido de alumnos, los cuales estaban juntos en una misma aula con un maestro. Estos no estaban formados ni capacitados para enseñar en una misma aula a alumnos de diferentes edades. Esta tendencia siguió también durante la mayor parte del siglo XX, en un proceso continuo de degeneración y declive de la educación rural (Camarero, 2009; Soler, 2008; Viñao, 2004).

La Ley General de Educación de 1970 impulsó un proceso de concentración escolar. Los alumnos de las escuelas rurales no podían seguir su escolarización obligatoria en sus pueblos y debían continuar estudiando en los centros de la ciudad más cercana. Esta política educativa comportó que muchos pueblos quedaran sin escuelas o con centros incompletos en los niveles superiores. Los niños debían desplazarse en edades tempranas a la localidad más próxima con escuela, aglutinando infantes de todos los pueblos cercanos. En las aldeas no había niños la mayor parte del día. En los años 80 se cambió la política educativa y se potenció la escolarización obligatoria en el espacio rural. Con la llegada al gobierno de España del partido socialista se impulsa la escuela rural mediante el programa de educación compensatoria de 1983. También Cataluña recibe los primeros traspasos de enseñanza que hicieron posible aplicar una política educativa propia para el ámbito rural. Se crearon las zonas escolares rurales (ZER) ${ }^{2}$, principalmente para potenciar y desarrollar estas escuelas. Se pretendía evitar el tradicional aislamiento de los maestros fomentando la creación de redes para compartir actividades, recursos económicos y humanos. Se dinamizaron las escuelas rurales y los niños no se desplazaban obligatoriamente a las ciudades para estudiar la educación primaria. Se construyeron nuevas escuelas o reformaron las antiguas. Los maestros debían desplazarse a los pueblos para enseñar. Los niños quedaban instalados en sus entornos domésticos y se facilitaba la vida de las familias en su pueblo (Soler, 2008).

En los primeros años de la Democracia, a partir de los años 80 , se cambia radicalmente la tendencia y lo rural aumenta en prestigio y valoración social. El maestro de la escuela rural es el principal referente cultural y educativo de muchos pueblos, tiene una relevancia que otros no poseen en otros contextos urbanos (Bustos, 2009). Esta escuela progresivamente va adquiriendo relevancia social y pedagógica. Según la investigación de Burrial et alter (2008) los docentes valoran principalmente la personalización del aprendizaje, la proximidad de las relaciones entre los actores de la comunidad educativa y la baja ratio de alumnos en el aula; también, en el mismo estudio alertan los docentes en contextos urbanos sobre la masificación de las aulas y las dificultades para

\footnotetext{
${ }_{1}^{1}$ Para ejercer de maestro en las escuelas incompletas bastaba un simple certificado de aptitud y moralidad expedido por la Junta Local y validado por el gobernador de la provincia, según establece la Ley Moyano, en el artículo 181.

2 Para conservar las escuelas pequeñas de los pueblos, romper el tradicional aislamiento y mejorar la calidad de la educación, el Decret 87/1987 marca la creación de las Zones Escolars Rurals (ZER): El agrupamiento de las diferentes escuelas en zonas rurales no debe comportar la desaparición de las mismas, tampoco romper su arraigo con el pueblo sino potenciarlas mediante la coordinación tecnicopedagógica y organizativa para juntarlas e estimularlas en un entorno social y cultural más amplio y estimulante de su misma entidad municipal (traducción del catalán).
} 
gestionar los conflictos. Siguiendo con Burrial et alter (2008), los maestros de la escuela rural consideran como factor de aprendizaje tener en el aula alumnos de diferentes edades y destacan tener en general una relación cercana y correcta con las familias y la comunidad. Además, las percepciones de los mismos enseñantes hacia las ZER son positivas o muy positivas en un 3,57 sobre 4 de los encuestados.

En los últimos años se han construido nuevas y modernas infraestructuras, carreteras y vías rápidas entre localidades. Los pueblos han dejado mayoritariamente de estar alejados y aislados y se han aproximado a las tendencias urbanas. Se han dotado de recursos tecnológicos... los centros educativos, también las escuelas rurales. Tradicionalmente esta escuela quedaba en el olvido y no se consideraba. En estos últimos años los diferentes gobiernos no han establecido diferencias relevantes entre centros ubicados en entornos urbanos y rurales. Se ha pretendido aplicar políticas educativas correctoras para fomentar y favorecer en lo posible la igualdad de oportunidades de todos los alumnos. Los pueblos y sus escuelas se han modernizado y urbanizado en los últimos tiempos (Aldomà, 2009).

La escuela rural se la dota de enseñantes suficientes y preparados, recursos materiales, los maestros tienen flexibilidad en el aula para transmitir conocimientos adaptados del entorno...; en muchas universidades se introduce en los planes de estudios del Grado de Educación Infantil y Primaria la materia de Escuela rural ${ }^{3}$. Los futuros maestros conocen más esta realidad y muchos realizan sus prácticas universitarias en estas escuelas. Según Estadella y Carrasco (1998) surge la convicción social que la vida en el pueblo y su escuela son dignas de conservar, dignificar y mejorar en lo posible. Aquella ruralidad tradicional, homogénea y conservadora se ha transformado hacia modalidades más plurales, complejas, dinámicas y flexibles; aunque todavía la perspectiva urbana tiende a representar lo rural como lo natural, armonioso y tranquilo. Siguiendo en la misma línea, Boix (2016) considera que la escuela rural y sus alumnos en la actualidad tienen las mismas posibilidades que aquellos residentes en entornos urbanos. Tradicionalmente las mejores familias del pueblo llevaban a sus hijos a las escuelas de la ciudad, estas tenían más reconocimiento. La escuela rural estaba reservada principalmente a los hijos de los campesinos e hijos de familias con escasas aspiraciones futuras. Con los cambios acaecidos en los años 80 y 90 la escuela rural se transforma y aumenta su prestigio.

Según Tahull (2016) la sociedad y la educación en los países desarrollados han tenido en los últimos años una transformación rápida e intensa, cambiando rápidamente estructuras, metodologías, procesos, dinámicas... En la sociedad y la escuela se ha instalado la complejidad, la pluralidad, el cambio permanente, la pérdida de referentes... Para Coll (2016) en este contexto social, cultural y educativo la personalización en la educación es la única posibilidad de formar, educar y capacitar a los alumnos en unas competencias educativas necesarias para vivir en sociedades postmodernas complejas. La escuela rural fundamentalmente por la baja ratio de alumnado tiene más posibilidades de personalizar, innovar y adaptar conocimientos.

Domingo (2014) y Feu (2008) destacan la revalorización de lo rural en los últimos años, en muchos pueblos ha habido un aumento demográfico destacado. La mejora de las infraestructuras, carreteras... ha facilitado la llegada de familias no originarias. Muchas escuelas rurales han incrementado la matriculación de alumnos y algunos centros se han ampliado. Además señalar el aumento de alumnos de origen extranjero. En muchos pueblos ha desaparecido el fantasma de cerrar obligatoriamente la escuela por no tener alumnos. Las autoridades han prohibido la matriculación de niños no residentes en la localidad por la excesiva demanda y la posible pérdida de las señas identitarias de estos centros. Situación que anteriormente era permitida y consentida. Desde los servicios territoriales del Departament d'Ensenyament se han limitado las plazas de la escuela rural a los residentes del pueblo, estos deben estar empadronados ${ }^{4}$.

\footnotetext{
${ }^{3}$ La Universidad de Lleida realiza la materia optativa de "Escuela y territorio: la escuela rural".

${ }^{4}$ Realizada la consulta con Inspección Educativa, se justifica la prohibición con el siguiente artículo de la Ley Orgánica 2/2006, de 3 de mayo, de Educación, en el artículo 81 sobre la "escolarización", en el punto 3 expone: En la educación Primaria, las Administraciones educativas garantizarán a todos los alumnos un puesto escolar gratuito en su propio municipio. Además, en el artículo 82, sobre "Igualdad de oportunidades en el mundo rural", en el punto 1 legisla: Las Administraciones educativas
} 
El artículo analiza la escuela rural de Cataluña en la actualidad. Se presenta información cuantitativa de los resultados académicos de los alumnos de $6^{\circ}$ de Primaria y se comparan con el global de Cataluña en diferentes materias; también datos sobre características de las escuelas rurales relacionándolas con otras organizaciones educativas; además el número de los alumnos matriculados en estos centros. Esta información se complementa con las aportaciones cualitativas de maestros, padres y exalumnos de estos centros, los cuales reflexionan sobre sus experiencias y vivencias sobre el tema tratado. Tal como explica Samper (2016) la ruralidad y sus escuelas demasiadas veces han sido estudiadas desde el mito y la ambivalencia. Muchas veces han sido analizadas desde perspectivas ideológicas esencialistas y valoraciones subjetivas; lejos de la investigación objetiva para comprender su realidad social, educativa y cultural. Esta investigación analiza la escuela rural a partir de datos de organismos públicos y entrevistas en profundidad a sujetos significativos y conocedores de primera mano de la vida en el pueblo y su escuela.

\section{Metodología}

Para la realización de la investigación se han utilizado diferentes técnicas, se ha seguido un método mixto para compilar datos significativos cuantitativos y cualitativos. Para obtener la información numérica se ha buscado en organismos oficiales, en el Instituto Nacional de Estadística de Cataluña y el Departament d'Ensenyament de la Generalitat de Cataluña sobre centros y alumnos de las escuelas rurales; además se han consultado los estudios del Consell Superior d'Avaluació de Cataluña sobre la puntuación global en las competencias básicas (2014-2016) de la Comunidad Autónoma y las zonas escolares rurales (ZER). A partir de los gráficos presentados se intenta comprender la significación micro de los resultados mediante diferentes entrevistas significativas a maestras/os, padres/madres y exalumnos/as todos conocedores de la escuela rural. La distribución de los participantes se realizó siguiendo diferentes criterios: de las cuatro provincias catalanas (Lleida, Tarragona, Girona y Barcelona), hombre/mujer, diferentes edades de los docentes y conocedores de la vida de los pueblos y la escuela rural. Todos los informantes son anónimos, personas con una experiencia significativa para el estudio, no aparecen en los medios de comunicación ni tienen una relevancia pública destacada.

Los datos cualitativos complementan la información cuantitativa, permiten comprender narrativamente y en profundidad las motivaciones y causas concretas de los gráficos desde la perspectiva de los propios sujetos. Se han buscado informantes que pudieran presentar una mirada concreta y profunda del objeto abordado. Son individuos de las cuatro provincias catalanas y anónimos. Se ha contactado inicialmente con conocidos del autor de la investigación y a partir de ellos se han buscado personas interesadas en colaborar. Se ha insistido que fuesen conocedores de la vida de los pueblos y la escuela rural. Todos los informantes tienen unas vivencias y experiencias vitales concretas, todas valiosas e interesantes. Comas d'Argemir (1992) afirma que todos los estudios sociales tienen la dificultad intrínseca de la subjetividad. Siempre se interpreta y describe la realidad social a partir de la propia mirada y singularidad. No debe interpretarse como una limitación o parcialidad, al contrario, son las reflexiones de actores relevantes de la comunidad educativa rural. Sujetos que han vivido en un entorno concreto y son conocedores en profundidad de una realidad educativa, social y cultural específica. En todo caso, no todas las aportaciones tienen el mismo valor, el investigador triangula, contrasta y compara los datos para construir el relato (Ander Egg, 2000).

Para la investigación se han realizado entrevistas a 8 maestras/os de Primaria, 7 padres/madres de alumnos y 10 exalumnos/as todos ellos con una relación y conocimiento específico de la escuela rural. Todas las/os maestras/os entrevistadas/os tienen más de 5 años de experiencia y uno de ellos con 27 años en la escuela rural. Son hombres y mujeres y están en centros de menos de 100 alumnos. Todos tienen entre 35 y 58 años de edad. 3 docentes son de la provincia de Lleida, 2 de Barcelona, 2 de Tarragona y 1 de Girona. En referencia a los padres/madres de los alumnos, todos

tendrán en cuenta el carácter particular de la escuela rural a fin de proporcionar los medios y sistemas organizativos necesarios para atender a sus necesidades específicas y garantizar la igualdad de oportunidades. La LOE garantiza una plaza escolar a todos los niños y asegura su matriculación en la escuela de su propio municipio. 
tienen como mínimo un hijo en la escuela rural en la actualidad. 3 madres/padres entrevistados son de la provincia de Lleida, 2 de Girona, 1 de Barcelona y 1 de Tarragona. En todos los casos la familia vive en la misma localidad de la escuela o están empadronados. Todos conocen en profundidad el centro educativo, son familias establecidas en la localidad y conocidas por los vecinos. En la mayoría de casos ellos mismos estudiaron en la misma escuela y conocen en profundidad sus características, particularidades y posibilidades educativas del centro en el pasado y la actualidad. También se han entrevistado 10 exalumnos/as de la escuela rural. En todos los casos son alumnos/as matriculados actualmente en la Universidad de Lleida, cursan diferentes grados, y son de diferentes localidades de la geografía catalana. 4 exalumnos son de la provincia de Barcelona, 3 de Lleida, 2 de Girona y 1 de Tarragona. Todos tienen entre 18 y 25 años de edad.

El proceso seguido para contactar con los informantes ha sido siempre el mismo. A partir de un contacto inicial con un conocido se le ha preguntado sobre posibles informantes interesados en colaborar en una investigación. Su participación ha sido desinteresada y exquisita, realizando aportaciones muy valiosas e interesantes y abriendo el marco de interpretación y valoración del objeto de estudio. Las entrevistas han sido abiertas, a partir de unos temas principales generales, se ha pretendido que los informantes aportaran sus experiencias, vivencias y reflexiones sobre la ruralidad y su escuela. El moderador, el autor de la investigación, iba preguntando sobre aspectos relevantes de la vida en el pueblo, la escuela, la vida en las aulas, los exámenes, metodologías, relación profesor-alumno, relaciones entre iguales... El entrevistador se ha adaptado a las necesidades del entrevistado y se han realizado las entrevistas en diferentes lugares: sus casas, bares y también en parques públicos. La duración ha sido entre sesenta y cien minutos aproximadamente. Se ha buscado la comodidad y tranquilidad de los colaboradores para propiciar su espontaneidad, libertad, extensión, detalle y profundidad del discurso. Se ha seguido el modelo de entrevistas abiertas descrito por Rubio y Varas (1999).

La información recogida ha sido amplia e interesante, aunque siempre limitada, y ha permitido tener una visión profunda del objeto de estudio. Todas las entrevistas se han grabado y las partes más destacadas se han transcrito por el autor. Los datos recogidos se ponderaron y equilibraron según las constantes y reiteraciones presentadas. La triangulación fue un elemento metodológico fundamental en la construcción del relato. La mayoría de la información obtenida no aparece en los resultados, aunque es igualmente relevante, ha servido para construir el relato y ponderar los datos. Todos los fragmentos transcritos en los resultados son significativos y representativos de las reflexiones de los informantes. Se ha decidido por un determinado texto por la fundamentación de los razonamientos y la claridad expositiva; además de estar en concordancia y sintetizar las opiniones y valoraciones de los otros entrevistados. Mills (1983) destaca que la construcción del relato exige un grado de imaginación sociológica para ordenar, dar sentido y coherencia al discurso como un todo.

\section{Resultados}

En este apartado se presentan datos cuantitativos y cualitativos de la escuela rural actual en Cataluña. Se muestran gráficos del total de municipios urbanos y rurales, escuelas y alumnos. También datos sobre la puntuación en las competencias básicas de $6^{\circ}$ de Primaria de los alumnos de los centros rurales. Para la realización del estudio se ha consultado diferentes fuentes documentales.

\subsection{Datos cuantitativos}

En la Figura 1 se contabiliza el total de municipios catalanes, distinguiendo entre aquellos con más y menos de 3.000 habitantes y la cantidad de escuelas rurales. 
Figura 1

Municipios catalanes según el número de habitantes

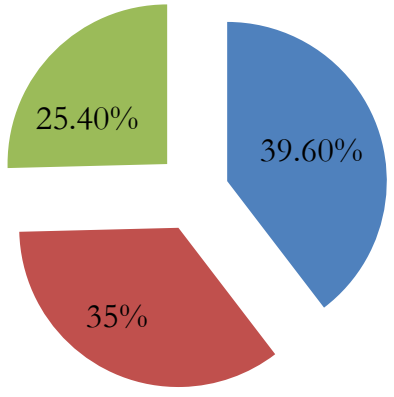

707 municipios no superan los 3.000

habitantes $(74,6 \%)$

De los 707 municipios que no superan los 3.000 habitantes, la escuela rural está en 332 pueblos $(35 \%)$

241 municipios superan los 3.000 habitantes $(25,4 \%)$

Fuente: Idescat. Institut d'Estadística de Cataluña. Datos de 2016.

Cataluña tiene en total 948 municipios, de los cuales 707 tienen menos de 3.000 habitantes, representa el 74,6\% del total, los otros tienen más población. Aquellos municipios que no superan los 3.000 habitantes, solamente tienen escuela rural 332 pueblos. En los otros casos, los niños deben necesariamente desplazarse a municipios próximos con escuela. En el siguiente gráfico se muestra comparativamente el número de todos los alumnos de Infantil, Primaria, Secundaria y la escuela rural.

Figura 2

Número de alumnos en la etapa Infantil y Primaria, Secundaria y Escuela Rural

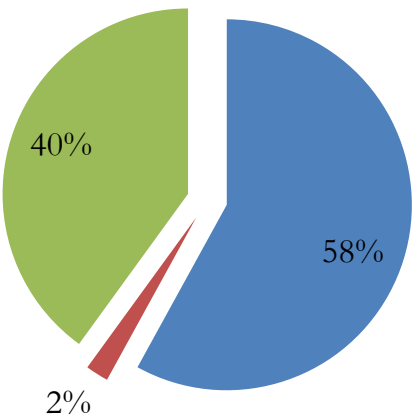

- Total de alumnos de Infantil y Primaria $702.307(58 \%)$

- Total de alumnos de la escuela rural 16.624 $(2 \%)$

Total de alumnos de Secundaria 602.815 $(40 \%)$

Fuente: Departament d'Ensenyament. Servei d'Indicadors i Estadística de la Generalitat de Cataluña. Curso 2015-2016.

En Cataluña hay un total de 1.305 .122 alumnos en la educación obligatoria. En Infantil y Primaria hay 702.307 alumnos, representa el 58\% del total de los alumnos; en Secundaria 602.815 alumnos, representa el $40 \%$ de los menores. La escuela rural tiene 16.624 alumnos, el $2 \%$ del total. Hay muchos municipios de menos de 3.000 habitantes, con pocas escuelas, habitantes y alumnos. La baja ratio permite al maestro personalizar e individualizar la enseñanza. En el siguiente gráfico se comparan los resultados académicos de las pruebas de competencias básicas de $6^{\circ}$ de Primaria entre el global de Cataluña y las escuelas rurales (ZER) por materias y en diferentes años. 
Figura 3

Competencias básicas de $6^{\circ}$ de primaria en tres años consecutivos en Cataluña

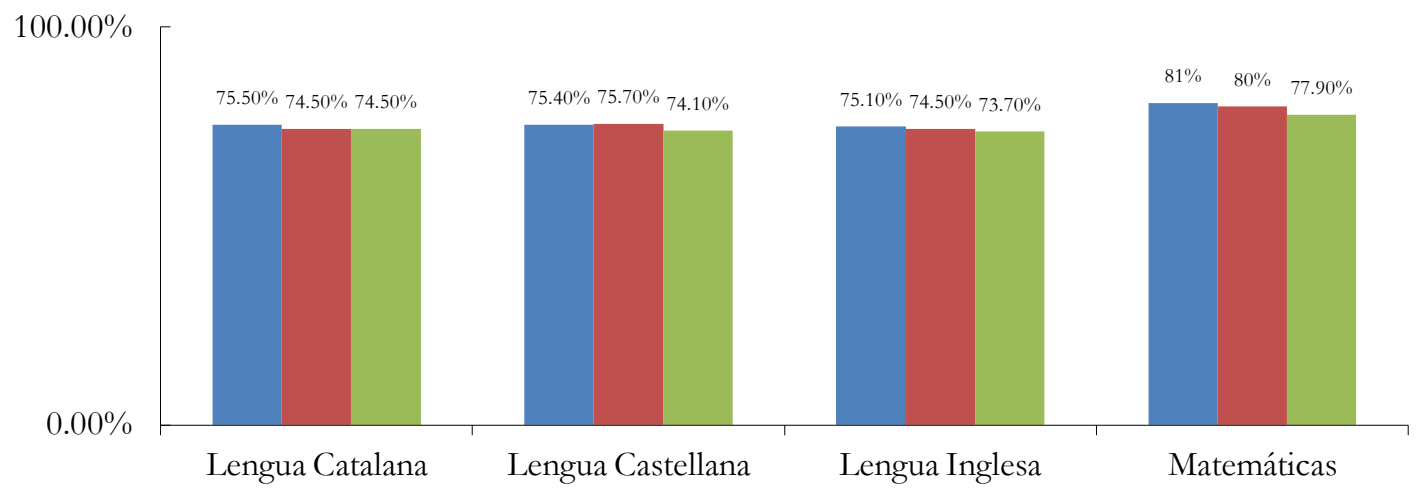

$\square 2014 \square 2015 \square 2016$

Fuente: Consell Superior d'Avaluación de la Generalitat de Cataluña (2017)

Figura 4

Competencias básicas de $6^{\circ}$ de primaria en tres años consecutivos en las zonas escolares rurales de Cataluña

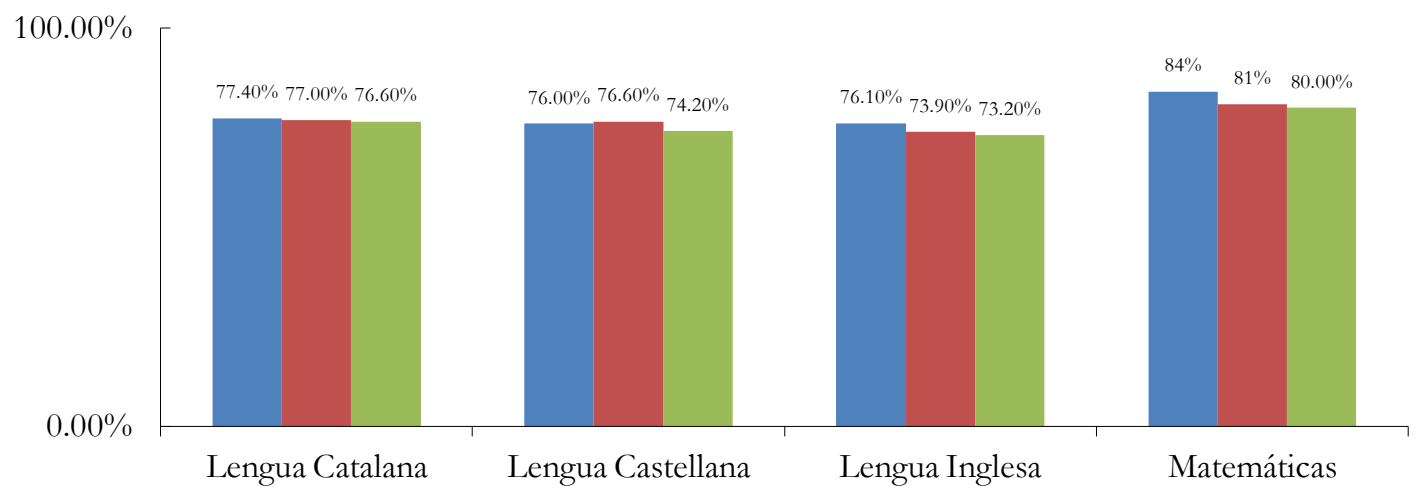

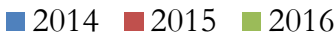

Fuente: Consell Superior d'Avaluación de la Generalitat de Cataluña (2017).

Las Firguas 3 y 4 presentan la distribución en diferentes materias (lengua catalana, castellana, inglesa y matemáticas) de las pruebas de competencias de $6^{\circ}$ de Primaria. En referencia a la lengua catalana, todos los años los resultados son mejores en las escuelas rurales respecto el global de Cataluña de forma significativa; en el año 2015 la diferencia es de 2,5\% superior en la escuela rural. Los otros años la diferencia también es importante, en más de $2 \%$. En lengua castellana también los resultados son mejores en las escuelas rurales, aunque no hay una diferencia tan destacada, varia del año 2014 en $0.6 \%$ al último año, el 2016, el 0,1\%. En lengua inglesa, en el año 2014 los alumnos de la escuela rural tenían mejores resultados, pero en los años 2015 y 2016 era superior la media global de Cataluña. En matemáticas las diferencias son destacadas, mejores en las escuelas rurales. En el año 2014 la diferencia es de 3,1\%, el 2015 de 0,9\% y el 2016 de 2,1\% respectivamente. Los datos muestran claramente que los alumnos de las escuelas rurales obtienen mejores resultados en todas las materias y años, a excepción de inglés en dos cursos. En lengua catalana y matemáticas las diferencias son significativas.

La escuela rural tiene unas características diferentes de la urbana. Permite la introducción dentro del aula de metodologías educativas innovadoras, un contacto cercano con las familias y alumnos, personalizar la educación... En el siguiente gráfico se pregunta a los maestros (por años de experiencia docente) sobre las peculiaridades de la escuela rural. 
Figura 5

Respuesta de los maestros, según año de antigüedad, sobre las peculiaridades de la escuela rural

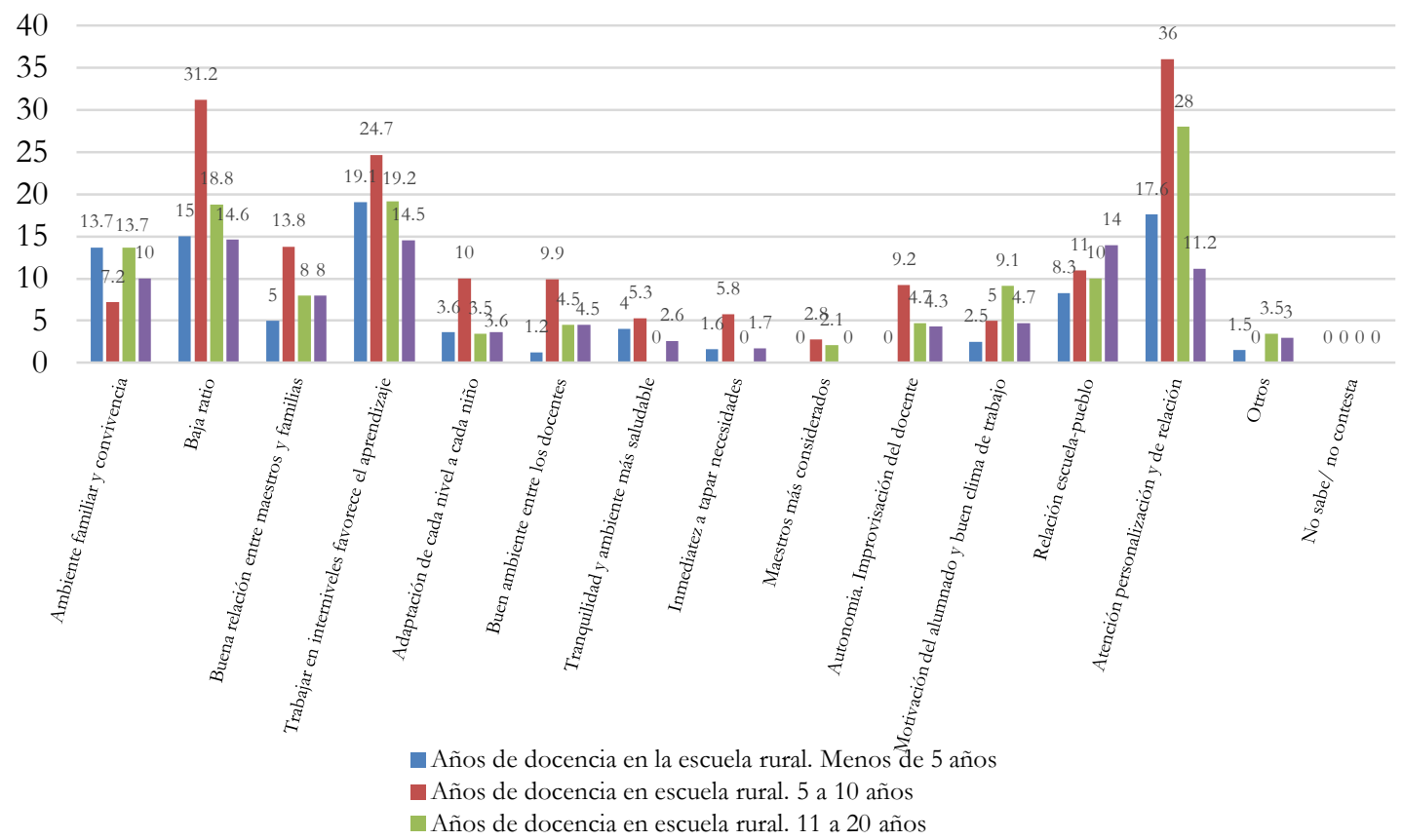

Fuente: Samper et al. $(2016,84)$.

La Figura 5 distingue entre maestros por años de docencia en la escuela rural, menos de 5 años, de 5 a 10 años, de 11 a 20 años y más de 20 años. Hay diferencias significativas entre las percepciones de los enseñantes en función de sus años de experiencia. En todo caso, destacan los docentes como elementos significativos de estos centros educativos: la atención personalizada y la relación entre los miembros de la comunidad educativa, la baja ratio en el aula y el trabajo y la relación entre alumnos de diferentes edades y niveles. En referencia a la atención personalizada son principalmente los maestros de 5 a 10 años de experiencia aquellos que valoran y consideran la individualización del aprendizaje; también aquellos de 11 a 20 años de experiencia. La baja ratio también es un elemento significativo y considerado por los maestros, principalmente entre aquellos de 5 a 10 años de experiencia. Permite conocer y trabajar mejor con los alumnos y su entorno. Además los enseñantes destacan las posibilidades de la escuela rural para favorecer intercambios y colaboración entre alumnos de diferentes edades y niveles educativos. En el gráfico se muestran algunos elementos significativos de la escuela rural y permite comprender sus mejores resultados académicos en $6^{\circ}$ de Primaria.

La información del Departament d'Ensenyament de la Generalitat de Cataluña no específica los datos por provincias, por titularidad de los centros educativos (privados, concertados, públicos...). Sería interesante poder comparar resultados académicos entre las escuelas rurales y diferentes tipologías de centros educativos. En todo caso, según los datos presentados, los alumnos de las escuelas rurales obtienen mejores resultados significativamente en las pruebas de $6^{\circ}$ de Primaria que la media de los alumnos catalanes. Además, destacar que estas mejores calificaciones académicas representan solamente el $2 \%$ del total de los alumnos catalanes.

\subsection{Datos cualitativos}

Presentados los datos cuantitativos globales de la escuela rural en Cataluña se requieren aportaciones cualitativas de informantes conocedores de la realidad social y educativa rural. Se presentan diferentes aportaciones de padres-madres, ex-alumnos y maestros sabedores desde diferentes puntos de vista de esta escuela. Los entrevistados son de diferentes zonas geográficas de Cataluña y permiten tener una mirada amplia y profunda de la ruralidad. 
Seguidamente se presentan las aportaciones de los padres de los alumnos de las escuelas rurales, los cuales reflexionan sobre las especificidades de los centros y la educación de sus hijos. Tienen la mirada de adulto y de padre-madre que siempre quieren la mejor educación posible para sus hijos. Las familias son una pieza fundamental de la comunidad educativa y elemento relavante para comprender dinámicas, situaciones y valoraciones de la escuela y del maestro. Resultan relevantes las aportaciones de Antonio de 40 años y padre de dos niños de 7 y 9 años, sus hijos están matriculados en una escuela rural de un pueblo (500 habitantes aproximadamente) de la provincia de Girona, dice:

"Para nosotros la escuela rural es ideal. Todos los niños del pueblo y de las masias cercanas van a la misma escuela. Todos nos conocemos, también a sus padres. Todos vamos juntos, en la escuela, pero también fuera. Por ejemplo en la Fiesta Mayor, los sábados, domingos... Las familias y los niños siempre somos los mismos." (Entrevistado 1)

El informante explica que la escuela está incrustada en la vida social y cultural del pueblo en un todo. Los niños, también las familias se conocen y siempre están juntos. Los habitantes participan en todas las actividades culturales y recreativas de la comunidad. Para comprender la escuela debe entenderse necesariamente la vida del pueblo. Antonio manifiesta que la escuela rural es ideal. Interpretamos su afirmación como un continuo de su vida satisfactoria en la comunidad trasladada a la educación de sus hijos. Las relaciones entre vecinos son buenas, relaciones de confianza, tranquilidad y contacto con la naturaleza. Estadella y Carrasco (1998) destacan que la vida en los pueblos continua siendo más tranquila y de mejor calidad que la de las ciudades. Se establecen lazos de solidaridad y reciprocidad con más frecuencia entre los vecinos. El informante no lo explica pero seguramente también hay conflictos. Familias e individuos enfrentados por diversos temas pero el entrevistado no hace referencia a estas situaciones. En todo caso, en el pueblo se conocen los vecinos, saben como son y como actuar. La vida en el pueblo permite superar estados de aislamiento y anomia de los sujetos propios de las urbes postmodernas (Aldomà, 2009).

Clara, madre de una niña de 10 años, la familia vive en un pueblo de Lleida de unos 800 habitantes aproximadamente, lleva su hija a la escuela rural del pueblo. Esta informante matiza las aportaciones de Antonio y señala algunas limitaciones de la vida rural:

"Para nosotras el pueblo a veces se queda un poco pequeño, faltan recursos y la escuela se queda pequeña. Siempre estamos los mismos en todos los lugares. A veces también encontramos a faltar gente nueva, niños nuevos... A veces en los pueblos caemos en la rutina de hacer siempre lo mismo con los mismos. A veces se hace imprescindible salir del pueblo e ir a Lleida ciudad para ver cosas diferentes y que nadie te conozca. Aquí en el pueblo, todos nos conocemos, pero hay gente que hace más de 10 años que ni se habla. Se cruzan por la calle y ni se saludan.” (Entrevistada 2)

La entrevistada matiza las afirmaciones realizadas por el informante anterior. Los habitantes del pueblo se conocen pero las relaciones sociales seguramente no son tan próximas y cálidas. Los pueblos son pequeños y siempre son los mismos en todos los sitios y esto provoca rutina y falta de motivación para dinamizar las actividades. Para ella es imprescindible salir del pueblo e ir a la ciudad, ver espacios y situaciones diferentes: tiendas, calles, gente desconocida y pasar desapercibida. No sabemos las causas intrínsicas de estas afirmaciones pero Clara alerta sobre la vida en los pueblos no siempre es plácida y familiar. En el pueblo hay vecinos que se cruzan por la calle y ni se hablan, "hace más de 10 años que ni se hablan". Samper (2016) explica que la ruralidad y las escuelas rurales se han descrito demasiadas veces desde una perspectiva esencialista, señalando solamente los aspectos positivos; también se deben señalar los conflictos y limitaciones. Clara destaca que en el pueblo la gente se conoce, pero muchos vecinos no tienen relaciones ni contactos entre ellos. Estadella y Carrasco (1998) destacan que la vida en el pueblo no es un ente sólido y monolítico; también hay diversidad, complejidad y conflicto.

Yolanda de 45 años y madre de un niño de 6 años viven en un pueblo (poco menos de 1.000 habitantes) de la provincia de Lleida. Resulta interesante sus aportaciones por ser una familia recién llegada al pueblo residente anteriormente en una gran ciudad: 
"La vida en el pueblo tiene muchas ventajas respecto a la vida de una ciudad. Yo soy de Barcelona y hace poco que estoy viviendo en el pueblo y la verdad que no hay color. Creo que la mayoría de las cosas son ventajas para los adultos pero también para mi hijo. En el pueblo nos han acogido bien, conocemos y tenemos contacto con todos los padres de la clase de mi hijo. Hacemos muchas actividades con otros padres y niños del pueblo. Aquí en poco tiempo conoces como funcionan las cosas y te adaptas. Todo es más fácil que en una gran ciudad, como Barcelona o Lleida. El mío todavía es pequeño, pero por lo que veo, cuando sean mayores siempre quedan y se hacen un grupo de amigos. Creo que los niños en los pueblos se relacionan más con otros, tienen más facilidades para jugar e ir solos por la calle." (Entrevistada 3)

La informante recientemente se ha instalado al pueblo y explica pricipalmente ventajas respecto la vida de la ciudad. El ámbito rural tiene una dinámica propia que el recién llegado debe comprender y acceptar. No explica estas normas explícitas y tácitas, pero se deben asimilar y respetar. La vida en el pueblo es más fácil y accesible; todo está más próximo. Los niños se pueden relacionar más fácilmente entre ellos en un entorno más flexible. Domingo (2014) y Feu (2008) destacan el incremento demográfico de muchos pueblos en los últimos años, con la llegada de familias de otras zonas de Cataluña o familias de origen extrangero. Estas familias (con sus hijos) se han instalado e integrado en la vida de los pueblos, originando un aumento en la matrícula de la escuela. Soler (2008) destaca la importancia de la escuela rural para consolidar y facilitar la vida de las familias en los pueblos. Los niños quedan instalados en un entorno conocido, accesible y próximo.

Sergio de 50 años y padre de un niño de 8 años viven en un pueblo (de unos 1.500 habitantes aproximadamente) de la provincia de Tarragona reflexiona sobre las fortalezas de la escuela rural:

"Creo que la escuela rural son ventajas. Yo destacaría principalmente tres: Primero, el número de alumnos por clase, en la clase de mi hijo son 12, ¿dime cuántos niños son en cualquier aula de una ciudad, eh? Creo que esto es un lujo... Segundo, esto conlleva que los profesores puedan estar más con los niños y puedan trabajar más con ellos. También, la tercera ventaja, creo, por lo que veo y me dice mi niño, puede haber una mejor relación entre los niños. Antes muchos padres de la ciudad llevaban a sus hijos a la escuela rural, ¡los llevaban ellos cada día! Entonces la Administración se dio cuenta que se estaba inflando la escuela rural de forma artificial y lo prohibieron. Ahora solamente pueden ir a la escuela rural los vecinos del pueblo, pero los de la ciudad no pueden. Entonces muchas familias se compraron una casa en el pueblo y se empadronaron para ir a la escuela." (Entrevistada 4)

Sergio valora las cualidades de la escuela rural y destaca principalmente la ratio de alumnos en el aula, siempre inferior a una escuela de la ciudad, pudiendo en algunos casos duplicar o triplicar. Considera la ratio "un lujo", unas condiciones que favorecen una educación de más calidad y personalizada. Los maestros pueden estar más tiempo con cada alumno y poder gestionar mejor las dinámicas y conflictos de clase. El clima emocional en el aula de la escuela rural es más adecuado y estimulante. Hay una mejor relación entre iguales y los docentes pueden gestionar mejor el grupoclase. Muchos padres residentes en otras localidades llevaban a sus hijos cada día a la escuela rural, hasta que la Administración lo prohibió. Para poder matricular a los niños en la escuela rural los padres deben estar empadronados en la población. Coll (2016) señala la importancia de la personalización del aprendizaje. En una sociedad compleja y plural los maestros deben necesariamente personalizar, innovar y adaptar conocimientos a los alumnos.

Presentadas las reflexiones de los padres-madres, se sigue con las aportaciones de los exalumnos de la escuela rural, los cuales valoran sus experiencias y vivencias de su educación Primaria. Se debe tener en cuenta que en la mayoría de casos han pasado unos 10 años de su experiencia, pero también resulta interesante la valoración y reflexión adulta realizada a posteriori. Todos los entrevistados son alumnos universitarios en la actualidad. Permite tener la mirada de unos jóvenes universitarios que han estudiado en la escuela rural. Anaïs de 22 años de un pueblo de Barcelona explica:

"Yo estuve en una escuela rural pequeñita del Montseny, en clase éramos 10 y siempre estábamos todos 
juntos. También recuerdo especialmente las maestras, eran muy cariñosas con nosotros. Las sentíamos muy cerca, eran como de la familia. Les teníamos mucha confianza y no creo que en una escuela más grande esto pase. La pena fue cuando tuvimos 12 años y tuvimos que ir a la capital de comarca a hacer ESO y fue muy diferente. No conocíamos a nadie y los profesores eran otra cosa. Creo que alguno ni nos conocía, ni sabían nuestro nombre. Yo recuerdo especialmente de la escuela rural como si fuese una gran familia, todos los niños y las maestras juntas. Recuerdo aquellos años con mucho cariño. Creo que todos tenemos estos sentimientos.” (Entrevistada 5)

La joven recuerda aquellos años en la escuela rural con especial cariño. Considera que las maestras eran especialmente cariñosas y próximas a los alumnos. Las relaciones sociales eran amistosas y muy individualizadas. No lo dice explícitamente, pero tener una baja ratio de alumnos en el centro facilitaba dinámicas sociales personalizadas y empáticas. La escuela era como una familia, estaban todos unidos (docentes y alumnos) en un proyecto común. Se establecían lazos de solidaridad y confianza mutua. Las escuelas rurales son diferentes de las escuelas urbanas, no justifica claramente la afirmación, se intuye principalmente sobre las características de las interacciones sociales. En los centros urbanos son más impersonales. Anaïs, al inicio de la ESO, tuvo dificultades para integrarse al nuevo centro educativo. Las relaciones sociales entre profesores y alumnos eran más distantes y frías y algunos "ni nos conocían, ni sabían nuestro nombre". Recuerda aquellos años de la escuela rural "con mucho cariño" y considera que todos los exalumnos tienen la misma opinión. Burrial et alter (2008) destacan en su estudio la buena relación en general de la escuela (maestros-alumnos) con las familias y la comunidad. En la misma investigación destaca la valoración positiva o muy positiva de los maestros hacia la escuela rural en un 3,57 sobre 4 de los encuestados. Las relaciones entre los actores de la comunidad educativa son más próximas e individualizadas.

Arnau de 18 años de un pueblo de Lleida explica la organización metodológica en su escuela, detalla la forma como aprendían:

"Estuve en una escuela rural con 8 niños en clase. Muchas veces pasábamos a buscarnos por casa los unos a los otros para ir a la escuela. Las clases eran flexibles y aprendíamos entre todos. Me acuerdo que yo aprendí muy rápido las tablas de multiplicar y a hacer las operaciones. Las repetían en clase los más grandes y los pequeños nos las acabábamos aprendiendo también. Muchas veces cuando los grandes acababan los ejercicios ayudaban a los más pequeños y hacían de maestros o se ponía a leer o a hacer leer a los más pequeños. A veces jugábamos todos a hacer construcciones o puzles dentro de la clase, cuando acabábamos nuestras actividades; cómo éramos pocos avanzábamos muy rápido los libros y teníamos tiempo para realizar otras cosas que supongo en clases con más niños no se podían hacer." (Entrevistada 6)

Según el informante, la organización metodológica en "las clases eran flexibles y todos aprendían de todos". En el aula había alumnos de diferentes edades y niveles educativos distintos. Los alumnos menores escuchaban contenidos de los mayores y siempre aprendían conceptos de cursos superiores. Siempre estaban más avanzados en conocimientos que alumnos de su misma edad de centros urbanos. También los educados debían esforzarse para ser maestros y explicar contenidos a niños más pequeños o de su misma edad que tenían dificultades con la lección. Entender los contenidos y explicarlos exigía un esfuerzo que los consolidaba. Arnau destaca principalmente las clases entre alumnos de diferentes edades como hecho diferencial y específico de la escuela rural, "a veces jugábamos con ellos a hacer construcciones o puzles". Las clases tenían un dinamismo y flexibilidad que no se daba en los centros urbanos. Burrial et alter (2008) hacen referencia a las fortalezas de la escuela rural: trabajar en interniveles favorece el aprendizaje y hay más relaciones y más próximas entre alumnos y maestros; también la baja ratio de alumnos en el aula favorece realizar proyectos educativos innovadores. Además, en el gráfico 5 los docentes presentan los puntos fuertes de la escuela rural, muchos destacan poder trabajar con alumnos de diferentes niveles como aspecto que favorece el aprendizaje.

Ana de 23 años de un pueblo de Tarragona explica principalmente las evaluaciones y el aprendizaje en la escuela rural. También se centra principalmente en la organización y gestión del aula por parte del docente: 
"Fui a una escuela rural de la provincia de Tarragona. La maestra nos conocía muy bien a todos y sabía lo que podía pedir a cada uno. Cuando pasé a la ESO era diferente, fui a un instituto de una ciudad cercana, éramos muchos en clase. Al principio estaba asustada porque no conocía a nadie. Al final me fue muy bien, pienso que salí muy bien preparada de la escuela rural. Sabía organizarme el trabajo y los exámenes, eso lo aprendí en la Primaria, como estábamos todos los cursos mezclados teníamos que ser bastante autónomos. También a veces explicábamos a los pequeños cuando no entendían alguna cosa. A mí me gustaba explicarles las lecciones de matemáticas, naturales... porque así yo también aprendía. La maestra también nos hacía corregir los ejercicios de los pequeños o entre nosotros. Ahora estoy estudiando el Grado de Primaria en la Universidad y nos explican metodologías novedosas y muy actuales que ya aplicaban los maestros en la escuela rural cuando yo estaba. Pienso que la escuela rural me ha dado estrategias como persona, alumna y futura maestra también.” (Entrevistada 7)

La informante valora buena la formación académica y humana recibida en la escuela rural. La llegada al instituto fue difícil, estaba asustada, no tenía muchas amigas y no conocía las dinámicas y formas de hacer de la ESO. Al poco tiempo se dio cuenta que tenía unos buenos fundamentos, "sabía organizarme el trabajo y los exámenes, eso lo aprendí en la Primaria, como estábamos todos los cursos mezclados teníamos que ser bastante autónomos". En Primaria recibió una buena formación y durante la Secundaria todo le fue más fácil, "fue muy bien, pienso que salí muy bien preparada de la escuela rural". En los gráficos 3 y 4 se comparan los resultados de las pruebas de competencias de $6^{\circ}$ de Primaria entre el global de Cataluña y las escuelas rurales. En casi todas las materias y años los resultados son superiores para las escuelas pequeñas. Actualmente, la entrevistada está matriculada en el Grado de Primaria en la Universidad y los profesores explican metodologías educativas innovadoras que coinciden con las formas de trabajar de la escuela rural de entonces. Muchos maestros usaban metodologías dinámicas y flexibles dentro del aula. Aldomà (2009) destaca la modernización y dignificación de pueblos y escuelas rurales. En estos centros se han introducido las mismas tecnologías de la información que en entornos urbanos. Se han implementado políticas para promover la igualdad de oportunidades de los alumnos de los centros rurales. Boix (2016) considera que esta escuela y sus alumnos actualmente tienen las mismas posibilidades que aquellos residentes en entornos urbanos. En la Figura 5 se destaca como punto fuerte de la escuela rural principalmente las posibilidades de personalizar el aprendizaje y tener una relación más próxima y familiar con los alumnos y sus familias. Se pueden establecer más fácilmente lazos de confianza y solidaridad mutua.

Finalizadas las aportaciones de los exalumnos, se introducen las reflexiones de los maestros, son profesionales de la docencia en activo e implicados directamente en las cuestiones de la vida de la escuela y el pueblo. Son los responsables de gestionar el centro educativo y enseñar a los alumnos. Son imprescindibles sus aportaciones y reflexiones para ponderar, valorar y situar el estado actual del tema tratado.

Marta de 49 años con 18 años de experiencia docente en la escuela rural, maestra de Primaria en un pueblo de menos de 1.000 habitantes de la provincia de Barcelona:

"Llevo muchos años trabajando aquí. Para mí hay muchas cosas positivas, pero quizás lo que más destacaría sería la proximidad con las familias. Conoces a todos los miembros de la familia y cómo estos responderán delante de situaciones escolares. Cuando tienes que hablar con ellos, puedes hacerlo en cualquier momento a la entrada o salida de la escuela. Al haber pocos niños y pocas familias existe mucha aproximación que en escuelas grandes no puedes mantener. Las familias en general en la escuela rural se preocupan más por el ámbito escolar, supongo que es por la accesibilidad de poder entrar y salir de la escuela o en cualquier momento poder hablar con el tutor o con cualquier especialista. También intervienen muchas veces en clase. Si estamos hablando del cuerpo humano y sabemos que hay un padre o una madre médico, le pedimos que nos venga a hablar del tema y lo hacen y así con muchas cosas. Las abuelas nos vienen a hacer las castañas y boniatos para la Castañada y también por Pascua nos hacen las monas dentro de la clase, explicando a los niños los ingredientes y cómo lo hacen. Los niños también participan en esta realización. En escuelas grandes esto no se podría hacer. A veces salimos por el pueblo a hacer trabajos por ejemplo entrevistas a la farmacéutica o a la carnicera o vamos a visitar alguna tienda de algún alumno y así muchas cosas." (Entrevistada 8)

La maestra explica la relación entre los maestros, padres y niños. Expone interacciones 
constantes y multidireccionales entre todos los sectores de la comunidad educativa. Los docentes conocen bien los padres y familiares de los alumnos; tienen un contacto continuo, directo y accesible. Cada día en las entradas y salidas de la escuela hablan padres y maestros sobre el niño; de cualquier situación significativa de la jornada. También los padres y abuelos entran con más facilidad y confianza dentro de la escuela, participan más estrechamente de la cotidianidad educativa, por ejemplo en la Castañada, Pascua... Según la maestra las familias se interesan más por la escuela, participan y están más dispuestas a colaborar en las actividades planteadas. Se establece una relación más estrecha entre la comunidad y la escuela y hay más confianza entre los diferentes sectores implicados en la educación. El gráfico 5 corrobora las reflexiones de la maestra. Los docentes tienen unas relaciones más cercanas y de confianza con la comunidad y las familias.

Miquel de 58 años con 27 años en la escuela rural, maestro de Primaria en un centro de la provincia de Girona:

"El clima en general es tranquilo, hay pocos alumnos y todo el mundo sabe lo que hay que hacer, la rutina está muy establecida, incluso en momentos de actividades más flexibles todos los alumnos saben lo que esperamos de ellos. Los pequeños lo van aprendiendo por imitación de los grandes. A la hora del patio que es donde puede haber más descontrol también está organizado, existen diferentes actividades para hacer. Pueden jugar en la pista a básquet o futbol, normalmente estos deportes los dejamos hacer dos veces por semana. Otro día hacemos juegos tradicionales, saltar a la cuerda, a la petanca... y así. Nosotros estamos por ciclos y lo que hacemos también durante la semana es que los alumnos del ciclo superior ayuden a leer a los del ciclo inicial. También los del ciclo medio explican cuentos a los de Infantil y al revés también. Todo es bastante más fácil que en una escuela grande, ya que movilizas menos niños y es más fácil de controlar. Supongo que en las escuelas que tienen 25 alumnos por clase o más no se atreven a que los alumnos salgan de sus clases para hacer actividades conjuntas, como hacemos nosotros. Bueno también hay escuelas rurales con muy pocos alumnos 7 u 8 en total y los niños no tienen posibilidades de relacionarse con niños de su edad. A lo mejor hay dos grandes y cuatro pequeños, entonces no creo que el ambiente sea tan rico. Más bien pobre, en estos casos la escuela rural, para mí no es tan aconsejable. No sé si llevaría a mi hijo entonces!" (Entrevistado 9)

El maestro con 27 años de experiencia docente en la escuela rural explica diferentes organizaciones educativas. Insiste principalmente en la flexibilidad de las actividades y las posibilidades de trabajo conjunto entre alumnos de diferentes niveles educativos, "los pequeños lo van aprendiendo por imitación de los grandes". También los niños mayores que saben leer enseñan a los pequeños que todavía no saben. Concluye afirmando que en la escuela rural, principalmente por la baja ratio de alumnado, es más fácil organizar actividades entre alumnos de diferentes niveles. En las escuelas más grandes, en las urbanas, todas estas organizaciones metodológicas son más complejas y difíciles de implementar en el aula. Miquel alerta también sobre los riesgos de tener pocos alumnos matriculados. Algunos tienen pocos niños, pueden ser "7 u 8 en total y los niños no tienen posibilidades de relacionarse con niños de su edad"; entonces la socialización y las relaciones entre iguales son muy pobres. En general la escuela rural es un privilegio pero en algunos centros con pocos alumnos matriculados está cuestionada, dice: "no sé si llevaría a mi hijo!" Considera fundamental la relación entre maestro y alumnos, también muy importante la relación horizontal entre los niños.

Joan de 38 años con 5 años de experiencia en la escuela rural de la provincia de Lleida como especialista de inglés:

“Antes estaba en una macro escuela, nada que ver con ésta. Lo que he notado es que en la escuela rural los alumnos tienen más tiempo para hablar en inglés. En una clase con muchos alumnos pregunto a tres o cuatro, pero aquí puedo preguntar a todos y saber si lo han aprendido y cómo lo han aprendido. Por ejemplo el día de antes les pongo una lista de palabras que se tienen que aprender o alguna frase hecha con la contestación y estas son el password que me tienen que decir para poder entrar en la clase el día siguiente, si no no entran. Aquí lo hago cada día, en una clase con 25 no lo podría hacer porque buena parte del tiempo de mi clase estaría haciéndolo. Con pocos alumnos es un momento y ellos están motivados. Pienso que pueden practicar el inglés oral en más ocasiones que si son muchos alumnos. También realizo actividades más motivadoras que con un número superior de alumnos no me plantearía. Otra de las ventajas es que conoces el nivel de aprendizaje de cada alumno a la perfección y también cómo 
puedes ayudar a cada uno dependiendo de lo que más necesita. También he notado que hago las clases más dinámicas porque al ser menos tengo que prepararme más actividades diferentes en una misma hora." (Entrevistado 10)

El maestro itinerante de inglés explica algunas de sus estrategias educativas; principalmente personaliza los contenidos y metodologías en el aula. Tiene tiempo para hablar con los alumnos, puede estar cerca de ellos y "saber si lo han aprendido y cómo lo han aprendido". Explica una práctica educativa que utiliza diariamente, cada día, al finalizar la clase el maestro les da una lista de palabras y frases hechas con la contestación que los alumnos deben aprenderse. Al día siguiente los alumnos deben saberlas y repetirlas para poder entrar en clase. Estas prácticas son muy motivadoras y los niños aprenden más vocabulario; con más alumnos en clase no podría trabajar de esta forma tan dinámica y personalizada. Joan considera que los maestros conocen mejor a los niños y las familias y pueden incidir más en su educación. En las Figuras 3 y 4 se muestran datos de los resultados globales de Cataluña y de las zonas escolares rurales (ZER). En todas las materias y años, excepción de dos años en inglés, los alumnos de las escuelas rurales obtienen mejores resultados académicos en las pruebas de competencias básicas de $6^{\circ}$ de Primaria.

Isabel de 43 años con 12 años en la escuela rural de un pueblo de la provincia de Tarragona hace referencia a alumnos con dislexia o TDAH:

“¿Y sobre el nivel educativo? Si creo que es superior en la escuela rural, simplemente porque conoces más a los alumnos y puedes dedicar más tiempo a los que les cuesta los aprendizajes y a los que necesitan más. Los exámenes acostumbran a no sorprenderte ya que durante las clases ya sabes el que lo va adquiriendo y el que tienes que ayudarle. Al ser pocos alumnos sabes el nivel de todos y también puedes adaptar los exámenes a las características de cada uno. Ahora nos encontramos mucho con niños con dislexia o TDAH que les tenemos que hacer alguna adaptación en los exámenes o los deberes. Cómo sabemos quiénes son ya lo hacemos. Creo que tenemos más vinculación afectiva que en escuelas donde tienes más alumnos por clase. Las pruebas diagnósticas de tercero y las de competencias básicas de sexto en esta escuela siempre salen por encima de la media de Cataluña y creo que es por lo que te he explicado antes. También creo que en una escuela grande las diferencias socioculturales de las familias son más diversas. En la escuela rural éste factor acostumbra a ser más homogéneo y eso dentro de la clase se agradece." (Entrevistada 11)

La maestra reflexiona principalmente sobre resultados educativos de los alumnos de la escuela rural. Su nivel educativo es superior a las escuelas urbanas, principalmente por la baja ratio de alumnos en clase. Los docentes pueden estar más tiempo con ellos y pueden personalizar más los contenidos; conocen mejor a los alumnos y "adaptan más los exámenes a las características de cada uno". En la escuela rural también hay niños con trastornos de aprendizaje como dislexia o TDAH, al tener una relación más cercana con los alumnos y las familias, el niño está más apoyado y comprendido. Según Isabel, en las pruebas diagnósticas de tercero y sexto de Primaria los resultados de la escuela rural son superiores a los centros urbanos. Estas pruebas son objetivas, realizadas en todos los centros educativos de Cataluña todos los años y coincide con los datos de las Figuras 3 y 4.

Los entrevistados y los datos cuantitativos presentan una escuela rural en la Cataluña actual lejos de ser tradicional, cerrada y atrasada como antes lo había sido. La percepción de los entrevistados y los gráficos muestran una escuela moderna y adaptada a los tiempos; con un dinamismo, flexibilidad y posibilidades que no tienen muchos centros urbanos. Los resultados académicos, contrastados con las pruebas de competencias básicas de sexto de Primaria, sitúan la escuela rural por encima de la media en Cataluña. Esta escuela y sus alumnos en la actualidad tienen las mismas posibilidades que los residentes en entornos urbanos. Con los cambios acaecidos se ha transformado y ha aumento significativamente su prestigio (Boix, 2016).

\section{Conclusiones}

En el artículo se han presentado datos cuantitativos significativos de la escuela rural de Cataluña. En esta Comunidad Autónoma hay en total 948 municipios, de los cuales 707 no superan los 3.000 
habitantes, son el 74,6\%, y 241 municipios superan los 3.000 habitantes, representan el 25,4\%. De los 707 municipios que no superan los 3.000 habitantes, la escuela rural está en 332 pueblos, el 35\%. Estos datos permiten situar aspectos demográficos significativos de Cataluña, específicamente del ámbito rural; son muchos pueblos con muy pocos habitantes distribuidos en todo el territorio. También, en total hay 1.305.122 alumnos catalanes en los diferentes niveles educativos, de los cuales solamente 16.624 niños están matriculados en la escuela rural, representa el $2 \%$ del total, según datos oficiales del Departament d'Ensenyament de la Generalitat de Cataluña en el año 2016.

En las pruebas de competencias básicas de $6^{\circ}$ de Primaria homogeneizadas en toda la Comunidad Autónoma los resultados académicos son superiores en casi todos los años y casi todas las materias en los centros rurales, en los años 2014, 2015 y 2016. En lengua catalana, castellana y matemáticas son superiores, dependiendo los años son claramente mejores, llegando en alguna materia y año hasta los tres puntos de diferencia. En lengua inglesa, en dos años, los resultados han sido inferiores en la escuela rural a la media de Cataluña, aunque por escaso margen.

La educación está cuestionada y en permanente reflexión en los últimos años por no haberse adaptado suficientemente a las transformaciones de las sociedades desarrolladas. Se cuestionan las metodologías educativas y las materias impartidas (Tahull, 2016). En esta investigación se aborda la escuela rural y presenta las reflexiones de diferentes personas de la comunidad educativa (maestros, padres y exalumnos). Estos manifiestan sentirse satisfechos o muy satisfechos por participar o haber tenido experiencias vitales en estos centros educativos. Una exalumna de la escuela rural, actualmente en la Universidad realizando el Grado de Primaria, manifiesta que algunos profesores universitarios explican nuevas metodologías educativas innovadoras que le recuerda sus años en Primaria. Se aprendía de la misma forma, principalmente personalizando contenidos y mediante metodologías dinámicas y flexibles (Coll, 2016). Según la investigación de Samper (2016) los maestros destacan positivamente de la escuela rural las posibilidades de personalizar la educación y la relación entre los diferentes agentes educativos, la baja ratio y las posibilidades de trabajar en el aula con niños de diferentes edades. Señalan principalmente el clima positivo dentro de clase y las buenas relaciones sociales entre maestros y niños. Ningún informante manifiesta situaciones conflictivas o un clima emocional negativo. La baja ratio de alumnos favorece una mejor gestión y control del aula. Los maestros están más cerca y conocen mejor a los alumnos. Miquel alerta del riesgo en algunos centros por los pocos niños matriculados. En algunas escuelas solamente son 6 o 7 educados y las relaciones sociales entre ellos pueden ser más pobres y menos estimulantes que en centros con más alumnos. El maestro reflexiona sobre la importancia de la relación entre enseñante y enseñado, más cercana y cálida; también de las interacciones sociales entre iguales.

A partir de las diferentes entrevistas a maestros, padres y exalumnos se muestra el grado de satisfacción y valoración de la escuela rural actual y pasada. Los informantes hacen referencia principalmente a las buenas relaciones entre maestros y alumnos, también destacan una mayor colaboración de los padres en las actividades organizadas en el centro educativo. Los docentes manifiestan conocer bien a los alumnos y padres y pueden personalizar mejor los itinerarios educativos y los contenidos; además tienen más posibilidades para realizar actividades estimulantes y motivadoras con los alumnos. Detectan antes y mejor las necesidades de los niños y pueden actuar conociendo mejor la situación concreta. Los maestros consideran que en la escuela rural están mejor integrados y socializados los niños con necesidades educativas de refuerzo o ampliación educativa (dislexia, TDAH, altas capacidades....). Algunos informantes destacan un ambiente tranquilo y saludable entre todos los actores de la comunidad educativa. Aldomà (2009) señala que la vida en los pueblos y su escuela se han modernizado y dignificado en los últimos años. Boix (2016) considera que la escuela rural y sus alumnos en la actualidad tienen las mismas posibilidades que aquellos residentes en entornos urbanos.

La escuela rural ha tenido en los últimos años una mejora significativa de los resultados académicos. Los diferentes agentes educativos valoran y respetan el trabajo realizado. Goza de buena salud y no tiene los mismos reproches y críticas que los centros urbanos. La escuela rural tiene una identidad propia, con unas características diferentes respecto las otras tipologías de escuelas. La organización está consolidada, tiene éxito, está valorada y poco cuestionada. Quizás las 
escuelas urbanas deberían incorporar aspectos metodológicos relevantes de las rurales. Tradicionalmente la escuela rural era una copia imperfecta y deformada de la urbana. Quizás la escuela de pueblo podría inspirar y ser modelo de los centros urbanos; ser faro que guie y oriente hacia una educación más dinámica, flexible y personal.

\section{Referencias}

Aldomà, I. (2009). Atles de la nova ruralitat. Lleida: Fundació del Món rural.

Ander Egg, E. (2000). Métodos y técnicas de investigación social. Buenos Aires: Lumen-Humanitas.

Boix, R. (2016). Factors de rendiment a l'escola rural catalana: característiques contextuals. Revista Catalana de Pedagogia, 10, pp. 59-74. Recuperado de: http://publicacions.iec.cat/repository/pdf/00000238/00000043.pdf.

Burrial, X., Garreta, J., Llevot, N., Navarro, P., Sala, T., \& Samper, LL. (2008). Mito y realidad de la escuela rural catalana. En Llevot, N., \& Garreta, J. (Coords.) Escuela ruraly sociedad (pp. 87-122). Lleida: Edicions de la Universitat de Lleida.

Bustos, J. (2009). La escuela rural española ante un contexto en transformación. Revista de Educación, 350, pp. 449-461. Recuperado de: http://www.revistaeducacion.mec.es/re350/re350_19.pdf.

Camarero, L. (Coord.). (2009). La población rural de España. De los desequilibrios a la sostenibilidad social. Barcelona: Obra Social de La Caixa.

Coll, C. (2016). La personalización del aprendizaje escolar. El qué, el por qué y el cómo de un reto insoslayable. En Vilalta, J.M. (Dir.). Reptes de l'educació a Catalunya. Anuari d'Educació 2015 (pp. 43104). Barcelona: Fundació Jaume Bofill.

Comas d'Argemir, D. (1992). La antropología social frente al análisis de problemas sociales en el propio contexto cultural. Papers d'Antropologia, 3. Tarragona: Arxiu d'Etnografia de Catalunya.

Consell Superior d'Avaluació de la Generalitat de Cataluña (2017). Resultados académicos de los alumnos de $6^{\circ}$ de Primaria en Cataluña Recuperado de: http://csda.gencat.cat/ca/arees_d_actuacio/avaluacions-consell/.

Departament d'Ensenyament. Servei d'Indicadors i Estadística de la Generalitat de Catalunya (2017). Total d'alumnes catalans. Curs 2015-2016. Recuperado de: http://ensenyament.gencat.cat/ca/departament/estadistiques/.

Domingo, L. (2014). Contribucions pedagògiques de l'escola rural. La inclusió a les aules multigrau. Un estudi de cas. Tesi doctoral de la Universitat de Vic. Recuperado de: http://www.tesisenred.net/bitstream/handle/10803/283165/tesdoc_a2014_domingo_laura_co ntribucions.pdf?sequence $=9$.

Estalella, H., \& Carrasco, S. (1998). La Catalunya rural contemporánea. En Giner, S. (Ed.). La societat catalana (pp. 367-376). Barcelona: Institut d'Estadística de Catalunya.

Feu, J. (2008). La escuela rural desde la atalaya educativa. En Llevot, N., \& Garreta, J. (Eds.) Escuela ruraly sociedad (pp. 61-86). Lleida: Edicions de la Universitat de Lleida.

IDESCAT (2017). Datos del total de municipios en Cataluña año 2016. Recuperado de: https://www.idescat.cat/?lang=es.

Llevot, N., \& Garreta, J. (2008). Escuela rural y sociedad. Lleida: Edicions de la Universitat de Lleida.

Rubio, M., \& Varas, J. (1999). Análisis de la realidad en la intervención social: métodos y técnicas de investigación. Madrid: CCS.

Samper, LL. (Coord.). (2016). Escoles, pobles i families. Noves perspectives sobre l'escola rural a Catalunya. Lleida: Edicions de la Universitat de Lleida.

Soler, J. (2008). El maestro y la fisonomía propia de la escuela rural. Una visión histórica de la escuela rural en Cataluña y España. En Llevot, N., \& Garreta, J. (Eds.) Escuela ruraly sociedad (pp. 11-41). Lleida: Edicions de la Universitat de Lleida.

Soler, J. (2005). L'escola rural a Catalunya al darrer quart de segle XX. De l'impacte de les polítiques educatives a la influencia de les transformacions socials, culturals i econòmiques: un primer balanç. Educació i Història: Revista d'Història de l'Educació, 8, pp. 103-131. Recuperado de: http://www.raco.cat/index.php/EducacioHistoria/article/view/223094/303898.

Tahull, J. (2016). La compleja transición de los adolescentes hacia la vida adulta. Revista de Antropologia Experimental, 16, pp. 27-44. doi: 10.17561/rae.v0i16.2853.

Viñao, A. (2004). Escuela para todos. Educación y modernidad en la España del s.XX. Madrid: Marcial Pons Historia. 